\title{
Reducing complexity: a visualisation of multimorbidity by combining disease clusters and triads
}

Ingmar Schäfer ${ }^{1 *}$, Hanna Kaduszkiewicz ${ }^{1,2}$, Hans-Otto Wagner ${ }^{1}$, Gerhard Schön $^{3}$, Martin Scherer ${ }^{1}$ and Hendrik van den Bussche ${ }^{1}$

\begin{abstract}
Background: Multimorbidity is highly prevalent in the elderly and relates to many adverse outcomes, such as higher mortality, increased disability and functional decline. Many studies tried to reduce the heterogeneity of multimorbidity by identifying multimorbidity clusters or disease combinations, however, the internal structure of multimorbidity clusters and the linking between disease combinations and clusters are still unknown. The aim of this study was to depict which diseases were associated with each other on person-level within the clusters and which ones were responsible for overlapping multimorbidity clusters.

Methods: The study analyses insurance claims data of the Gmünder ErsatzKasse from 2006 with 43,632 female and 54,987 male patients who were 65 years and older. The analyses are based on multimorbidity clusters from a previous study and combinations of three diseases ("triads") identified by observed/expected ratios $\geq 2$ and prevalence rates $\geq 1 \%$. In order to visualise a "disease network", an edgelist was extracted from these triads, which was analysed by network analysis and graphically linked to multimorbidity clusters.

Results: We found 57 relevant triads consisting of 31 chronic conditions with 200 disease associations ("edges") in females and 51 triads of 29 diseases with 174 edges in males. In the disease network, the cluster of cardiovascular and metabolic disorders comprised 12 of these conditions in females and 14 in males. The cluster of anxiety, depression, somatoform disorders, and pain consisted of 15 conditions in females and 12 in males.

Conclusions: We were able to show which diseases were associated with each other in our data set, to which clusters the diseases were assigned, and which diseases were responsible for overlapping clusters. The disease with the highest number of associations, and the most important mediator between diseases, was chronic low back pain. In females, depression was also associated with many other diseases. We found a multitude of associations between disorders of the metabolic syndrome of which hypertension was the most central disease. The most prominent bridges were between the metabolic syndrome and musculoskeletal disorders. Guideline developers might find our approach useful as a basis for discussing which comorbidity should be addressed.
\end{abstract}

Keywords: Multimorbidity, Multimorbidity patterns, Disease combinations, Epidemiology, Chronic diseases, Elderly people, Factor analysis, Network analysis, Observed-expected-ratios, Claims data set

\footnotetext{
* Correspondence: in.schaefer@uke.de

${ }^{1}$ Department of Primary Medical Care, University Medical Center

Hamburg-Eppendorf, Martinistr. 52, Hamburg 20246, Germany

Full list of author information is available at the end of the article
} 


\section{Background}

Multimorbidity is the presence of multiple chronic conditions that can include any type of disease combinations. For example, using a list of 46 chronic conditions, we found $99 \%$ of the 15,180 theoretically possible combinations of three diseases ("triads") in a large claims data set [1]. Over the last decade, many research groups have tried to understand the complexity of multimorbidity due to its high prevalence, estimated to affect 50\% to 99\% of the older population [2] and its association with adverse health outcomes, such as functional status decline, lower quality of life, higher mortality risk, increased health care utilisation and, therefore, rising health care costs [3,4]. Despite the many studies analysing the associations of diseases [5,6], it is still unknown how to reduce the complexity of multimorbidity, e.g. in order to permit a more thorough consideration of multimorbidity in clinical practice guidelines.

In multimorbidity research, it is often assumed that health outcomes are mainly influenced by the individual diseases themselves [7] and that there may be additional effects of disease interactions. A recent review identifies 14 studies that try to reduce the heterogeneity of multimorbidity [8], which can be done in two possible ways. First, one can start with the full complexity of multimorbidity and identify clusters of diseases that are often diagnosed together ("cluster method") [9]. Second, one can start with single diseases and group them into disease combinations usually based on prevalence figures ("combination method") [1].

Both methods have limitations. In using the combination method, the unit size (e.g. disease triads) does not necessarily match the number of conditions of a single person so that there are always more combinations than people in a data set. Regarding triads, for example, if a person has four diseases he/she will appear four times in the data set. The combination method does not show how combinations overlap in people, so that it is difficult to infer from combinationlevel to person-level. In contrast, using the cluster method, one cannot determine how diseases are associated within one cluster. For example, some diseases might only be in the same cluster because they are linked through a third disease, e.g. hypertension and renal insufficiency might be linked through diabetes. As clusters overlap, one might also ask which diseases serve as a bridge between clusters.

Therefore, this study aims at transcending the cluster and combination methods in order to depict which diseases are associated with each other on person-level within the clusters and which ones are responsible for the overlapping of multimorbidity clusters.

\section{Methods}

Data set

The analyses are based on ambulatory data of the Gmünder ErsatzKasse, a German statutory health insurance company with 1.7 million insurants (in 2008), which corresponds to $2.4 \%$ of the statutory insured population [10]. In Germany, about $90 \%$ of the total population is insured by statutory health insurance companies, because there is an obligation to be part of the statutory insurance system. The only exceptions are public servants ("Beamte"), clerics, professional soldiers, self-employed people (except artists and farmers), and people with an income over $52.000 € /$ year who decide that they want to leave the statutory health insurance system. People in Germany who are not part of the statutory insurance system can be insured by private health insurance companies.

The Gmünder ErsatzKasse has a greater proportion of elderly male insurants than the general population of Germany, therefore, data analyses have to be adjusted for gender. The dataset contains pseudonymised data from every insured member of this company. The sample used for our analyses consists of all people aged 65 years and older who were continuously insured throughout the year 2006. The claims data set includes all diseases reported by ambulatory physicians. All problems managed by physicians within the statutory health insurance system have to be coded in ICD-10 (International Statistical Classification of Diseases and Related Health Problems, 10th Revision) and forwarded to the health insurance companies as regulated by $\$ 295(1)$ of the German Social Security Code $S G B$ $V$ and $\$ 44(3)$ of the Federal Collective Agreement within the statutory health insurance system in Germany [11]. As there is no limit to the number of diagnoses per patient that can be forwarded to health insurance companies, all diagnoses by ambulatory physicians are included in the data set.

The analysis of morbidity was based on a list of 46 defined diagnosis groups of chronic diseases (see below) derived from ICD-10 codes. The diagnoses were only counted if they were coded in at least three out of four quarters (three month periods) in 2006. This criterion was chosen in order to increase the validity of the diagnoses based on claims data by avoiding transitory or even accidental diagnoses. Prevalence, gender-specific rank order, and ICD-10 codes of the diagnosis groups have been published in another study [9].

The methods for compiling the list of 46 diagnosis groups have been described elsewhere in detail [1]. In short, we used the most frequent conditions documented in GP surgeries as listed in a panel survey of the Central Research Institute of Statutory Ambulatory Health Care in Germany ("ADT-Panel") [12]. Chronicity of diagnoses was assessed using the report of the expert group on the implementation of a morbidity oriented risk adjustment scheme in the German Statutory Health Insurance [13]. In order to capture a comprehensive picture of the disease patterns in individual patients, we amended this list by all chronic conditions with a prevalence $\geq 1 \%$ in the age 
group $\geq 65$ years in the data set of the Gmünder ErsatzKasse in 2006. ICD-10 codes were grouped together if diseases and syndromes had comparable pathophysiological mechanisms and if ICD codes of related disorders were supposed to be used ambiguously by coding physicians in clinical reality, respectively.

\section{Statistical analyses}

The main feature of the statistical methods used in this study is a network analysis [14] which shows all associations between the selected diseases in graph format. The resulting "disease network" shows which diseases are often diagnosed together and how many associations to other diseases each disease has. The network analysis incorporates information from two previously published analyses: 1) triads identified by prevalence and observed/ expected ratio [1] and 2) multimorbidity clusters from factor analysis [9].

The analyses were conducted in the multimorbid population, i.e. we included all patients who had at least three chronic conditions from the list of 46 diagnosis groups. We performed our analyses for both genders separately, because there are big differences between males and females in the prevalence rates of chronic conditions and we, therefore, wanted to allow for a possibly different association structure. To determine which diseases were associated with each other, we started with triads, i.e. combinations of three diseases. We used the prevalence as a criterion for relevance. Triads with a prevalence $<1 \%$ in the data set were excluded. The association between diseases was determined by observed/expected ratios ("O/Eratios"). The expected prevalence of triads was calculated by multiplying the total prevalence of the single diseases within this triad by each other. The O/E-ratio was then estimated by dividing the observed by the expected prevalence of the triad. We defined diseases within a triad as associated with each other when the O/E-ratio was $\geq 2$. For example, if we looked at the combination of hypertension (prevalence in the multimorbid male population: 69.4\%), lipid metabolism disorders (48.0\%) and chronic low back pain (41.0\%), we expected a prevalence of $13.7 \%$ $(0.694 * 0.480 * 0.410=0.137)$. We observed a prevalence of $13.9 \%$ for this combination in the data set. Therefore, the $\mathrm{O} / \mathrm{E}$-ratio in this case was $1.01(0.139 / 0.137=1.01)$, which was below 2 and, therefore, the three diseases were not associated according to our definition and the triad was excluded from further analyses. The analyses for identifying relevant and associated triads were conducted using $\mathrm{R}$ version 3.0.1 [15].

For triads considered as relevant and associated, we extracted an edgelist, i.e. a list of all disease pairs contained within the selected triads. The edgelist was used to visualise the disease networks of both genders. The position of the diseases within the two-dimensional space was computed by a multidimensional scaling procedure. These analyses were conducted with the Stata 12.1 network analysis module Netplot by Rense Corton [14].

The results from this procedure were grouped (see next paragraph) using the multimorbidity clusters identified by factor analysis presented in a previous study [9]. In short, correlations between diagnosis groups were analysed by exploratory factor analysis based on a tetrachoric correlation matrix. We used an oblique (oblimin) rotation of factor loading matrices. The factors that resulted from this analysis could be interpreted as clusters of diagnosis groups frequently associated with each other. Factors were regarded as substantial if they had an eigenvalue of 1.0 or more. Diagnoses were assigned to a cluster if they had a factor loading of 0.25 or more on the pattern in charge.

As a next step of the analysis presented here, the single diseases were grouped into the multimorbidity clusters of "cardiovascular and metabolic disorders" and "anxiety, depression, somatoform disorders and pain". The pattern "neuropsychiatric disorders" was not used as a grouping variable because we did not find a triad that represented three diseases from this pattern in either gender. However, the single neuropsychiatric diseases were still part of the data analysis. The relation between diseases and the multimorbidity cluster they were assigned to was pictured in two figures based on the Netplot graphs mentioned above.

We also calculated quantitative measures for the disease networks, which included node degree centrality (i.e. the number of observed edges of one disease in relation to the number of possible edges, which was used as a measure for the connectedness of a disease) and node betweenness centrality (i.e. the number of times that diseases served as a bridge in the shortest pathway between other diseases, which was used as a measure for the potential influence of a disease on the distribution of other diseases in one person). Additionally, we also calculated the average node betweenness centrality as a (negative) measure for the total extent of accumulation of diseases within the networks. These analyses were performed by Visone 2.7.3.

In order to provide greater confidence in the study results, we replicated all analyses with the ambulatory data of the Gmünder ErsatzKasse from 2004. As the prevalence of most chronic conditions was significantly lower in 2004, we chose to lower the prevalence criterion for triads from $1.0 \%$ to $0.85 \%$ in this data set, which resulted in a comparable number of eligible triads as in 2006. All other methods, i.e. the methods for data preparation, factor analyses, extraction of the edgelists, and network analysis, as well as the O/E-ratio used for triad inclusion, were the same as in 2006.

\section{Ethics statement}

The research presented in this paper was conducted according to the principles expressed in the Declaration of 
Helsinki. We did not have to obtain informed consent because our research was based on insurance claims data and the data set was analysed anonymously (as regulated by German law pursuant to $\$ 75$ SGB X). The study was approved by the Ethics Committee of the Medical Association of Hamburg (approval no. PV3057). The approval included the waiver of consent.

\section{Results}

We found a total of 149,280 patients in our 2006 data set of which 98,619 (66.1\%) were defined as multimorbid because they had 3 or more chronic conditions from our list of 46 diagnosis groups. The distribution of age, gender, and the number of chronic conditions in the multimorbid population is shown in Table 1. 43,632 (44.2\%) of these patients were female and 54,987 (55.8\%) were male. The triads by multimorbidity clusters that had previously been identified by factor analysis are shown in Tables 2 and 3. We found 13,460 triads of chronic conditions in the female population of which 932 had a prevalence $\geq 1 \%$ and 57 of these also had an $\mathrm{O} / \mathrm{E}$ ratio $\geq 2$ (cf. Table 2). In the male population, we found 14,105 triads of which 830 had a prevalence $\geq 1 \%$ and 51 of these also had an O/E ratio $\geq 2$ (cf. Table 3). In total, the selected triads of females consisted of 31 chronic conditions with 200 edges and the selected triads of males included 29 chronic conditions with 174 edges.

The prevalence, the number of edges as well as degree and betweenness centrality of chronic conditions are shown in Table 4. In both genders, the condition with the highest number of edges was chronic low back pain (16 edges in females and males), i.e. chronic low back pain was associated with 16 other chronic conditions, which corresponded to $8.0 \%$ of all possible connections (degree centrality) in females and 9.2\% in males. Chronic low back pain also was the most central disease in the networks of both genders in terms of being the most important mediator of connections between other chronic conditions (betweenness centrality). Another disease with high betweenness centrality in both genders was hypertension. Depression also was an important mediator of disease connections in females, but not in males. The average level of accumulation in the female disease network (average node betweenness) was 3.2\%, while the male disease network had a slightly higher average node betweenness of $3.4 \%$ and thus was slightly less accumulated than the female disease network.

Figure 1 shows the disease associations in the multimorbid female population and Figure 2 shows them in the multimorbid male population. In these figures, each ellipse represents a chronic condition and the surface area of each ellipse is proportional to the prevalence of the disease. Each line represents an association between two chronic conditions. For example, the triad "hypertension + urinary incontinence + cancers" in the male population is represented by three ellipses and the three lines between these ellipses in the lower right corner of Figure 2. These figures can be interpreted that two diseases linked by an edge are often diagnosed together on person-level. For this interpretation, only direct links are relevant, e.g. in the female population, chronic low back pain is linked to somatoform disorders and dizziness, but there is no edge between somatoform disorders and dizziness. This means that chronic low back pain is often diagnosed together with a somatoform disorder, and chronic low back pain also is often diagnosed together with dizziness, but the diagnosis of dizziness does not often occur in conjunction with a somatoform disorder diagnosis.

Our replication analysis in the 2004 data set showed similar results as in 2006. A total of 123,224 patients were included in these analyses of which 72,548 were multimorbid according to our definition. Age, gender, and the number of chronic conditions of this population are shown in Table 1. Regarding the factor analysis, we found a very similar association structure in 2004 with the same three clusters extracted for males and females as in 2006. The only notable difference in the analyses presented here was that the ADS and pain cluster in the male population of 2004 did not include prostatic hyperplasia and sexual dysfunction. Instead, one additional multimorbidity cluster with prostatic cancer and related disorders had been extracted. Except for that, only minor differences between both years were found in the factor analyses.

In 2004 we found 957 triads with a prevalence $\geq 0.85 \%$ in the female population and 56 of these also had an $\mathrm{O} / \mathrm{E}$ ratio $\geq 2$. In the male population, we found 819 triads with a prevalence $\geq 0.85 \%$ and 49 of these also had an $\mathrm{O} / \mathrm{E}$ ratio $\geq 2$. In total, the selected triads of females consisted of 29 chronic conditions with 194 edges and the selected triads of males included 27 chronic conditions with

Table 1 Age and the number of diagnosis groups by gender in the multimorbid* population of 2006 and 2004

\begin{tabular}{lccccc}
\hline & \multicolumn{2}{c}{$\mathbf{2 0 0 6}$} & & \multicolumn{2}{c}{$\mathbf{2 0 0 4}$} \\
\cline { 2 - 3 } & Females & Males & & Females & Males \\
\hline Number of patients & $43,632(44.2 \%)$ & $54,987(55.8 \%)$ & & $32,369(44.6 \%)$ & $40,179(55.4 \%)$ \\
Age: mean $\pm s d$ & $73.3 \pm 6.8$ & $72.2 \pm 5.9$ & $73.6 \pm 6.8$ & $72.3 \pm 5.9$ \\
Chronic conditions: mean \pm sd & $6.0 \pm 2.7$ & $5.9 \pm 2.6$ & $5.7 \pm 2.6$ & $5.6 \pm 2.5$ \\
\hline
\end{tabular}

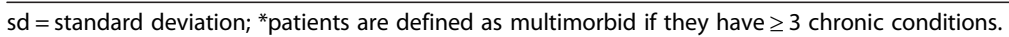


Table 2 Triads with a prevalence $\geq 1 \%$ and an observed/expected ratio $\geq 2$ by multimorbidity clusters in the female population with $\geq 3$ chronic conditions $(n=43.632)$

\begin{tabular}{l}
\hline Cardiovascular and metabolic disorders \\
\hline Diabetes mellitus + Hyperuricemia/Gout + Liver diseases \\
Diabetes mellitus + Obesity + Hyperuricemia/Gout \\
Hypertension + Hyperuricemia/Gout + Renal insufficiency \\
Chronic ischemic heart disease + Cardiac arrhythmias + Cardiac insufficiency \\
Lipid metabolism disorders + Hyperuricemia/Gout + Liver diseases \\
Diabetes mellitus + Chronic ischemic heart disease + Cardiac insufficiency \\
Diabetes mellitus + Chronic ischemic heart disease + Neuropathies \\
Lipid metabolism disorders + Cardiac arrhythmias + Cardiac valve disorders \\
Hypertension + Cardiac arrhythmias + Cardiac valve disorders \\
Diabetes mellitus + Chronic ischemic heart disease + Hyperuricemia/Gout \\
Severe vision reduction + Diabetes mellitus + Neuropathies \\
Diabetes mellitus + Chronic ischemic heart disease + Atherosclerosis/PAOD \\
Lipid metabolism disorders + Obesity + Hyperuricemia/Gout \\
Lipid metabolism disorders + Diabetes mellitus + Hyperuricemia/Gout \\
Hypertension + Obesity + Hyperuricemia/Gout \\
Hypertension + Hyperuricemia/Gout + Liver diseases
\end{tabular}

Lipid metabolism disorders + Chronic ischemic heart disease + Cardiac valve disorders O/E-ratio Prevalence in \%

Hypertension + Chronic ischemic heart disease + Renal insufficiency

Hypertension + Atherosclerosis/PAOD + Cerebral ischemia/Chronic stroke

Lipid metabolism disorders + Hyperuricemia/Gout + Cardiac insufficiency Hypertension + Chronic ischemic heart disease + Cardiac insufficiency

Diabetes mellitus + Chronic ischemic heart disease + Cardiac arrhythmias

Hypertension + Diabetes mellitus + Renal insufficiency

Diabetes mellitus + Chronic ischemic heart disease + Obesity

Lipid metabolism disorders + Obesity + Liver diseases

Lipid metabolism disorders + Hyperuricemia/Gout + Atherosclerosis/PAOD

Lipid metabolism disorders + Liver diseases + Atherosclerosis/PAOD

Hypertension + Diabetes mellitus + Obesity

Lipid metabolism disorders + Chronic ischemic heart disease + Hyperuricemia/Gout

Severe vision reduction + Diabetes mellitus + Atherosclerosis/PAOD

\section{Anxiety, depression, somatoform disorders and pain}

Chronic low back pain + Depression + Somatoform disorders

Chronic low back pain + Asthma/COPD + Allergies

Chronic low back pain + Intestinal diverticulosis + Chronic gastritis/GERD

Chronic low back pain + Depression + Insomnia

Joint arthrosis + Depression + Somatoform disorders

Chronic low back pain + Depression + Dizziness 
Table 2 Triads with a prevalence $\geq 1 \%$ and an observed/expected ratio $\geq 2$ by multimorbidity clusters in the female population with $\geq 3$ chronic conditions $(n=43.632)$ (Continued)

\begin{tabular}{lll}
\hline Chronic low back pain + Chronic gastritis/GERD + Insomnia & 2.1 & 1.1 \\
Chronic low back pain + Gynaecological problems + Somatoform disorders & 2.0 & 1.2 \\
Depression + Osteoporosis + Chronic gastritis/GERD & 2.0 & 1.1 \\
Both multimorbidity patterns & O/E & 2.5 \\
Joint arthrosis + Obesity + Hyperuricemia/Gout & 2.5 & 1.2 \\
Chronic ischemic heart disease + Lower limb varicosis + Cardiac insufficiency & 2.4 & 1.0 \\
Joint arthrosis + Chronic ischemic heart disease + Cardiac insufficiency & 2.3 & 1.5 \\
Chronic low back pain + Hyperuricemia/Gout + Liver diseases & 2.2 & 1.2 \\
Hypertension + Depression + Anxiety & 2.1 & 1.0 \\
Chronic low back pain + Obesity + Hyperuricemia/Gout & 2.1 & 1.5 \\
Diabetes mellitus + Obesity + Lower limb varicosis & 2.1 & 1.5 \\
Joint arthrosis + Diabetes mellitus + Neuropathies & 2.1 & 1.4 \\
Chronic low back pain + Depression + Neuropathies & 2.1 & 1.4 \\
Chronic low back pain + Neuropathies + Chronic gastritis/GERD & 2.0 & 1.2 \\
Joint arthrosis + Obesity + Lower limb varicosis & 2.0 & 2.0 \\
Lipid metabolism disorders + Asthma/COPD + Allergies & 1.1 \\
\hline
\end{tabular}

O/E: observed/expected ratio; PAOD: peripheral arterial occlusive disease; COPD: chronic obstructive pulmonary disease; GERD: gastroesophageal reflux disease.

164 edges. The edgelists of 2004 produced comparable figures of disease networks as in 2006 with a clear separation between the multimorbidity clusters: 1) ADS and pain and 2) cardiovascular and metabolic disorders. Degree and betweenness centrality in the cluster "ADS and pain" were comparable to 2006, as chronic low back pain also had the highest degree and betweenness centrality of all nodes and depression still had the second highest betweenness centrality in females in this cluster. The only notable differences related to betweenness centrality in the cluster "cardiovascular and metabolic disorders", as hypertension did not constitute an important mediator between chronic conditions in any gender, but was replaced in 2004 by hyperuricemia/gout in males and chronic ischemic heart disease in females.

\section{Discussion}

\section{Associations between diseases}

This is the first study that uses a systematic approach to integrate the two current methods to determine multimorbidity clusters in the older population and, therefore, includes information about the association between single diseases on person-level. It shows epidemiologically relevant associations between diseases in graph format and also gives information about the number of connections of one disease to other diseases as well as how much potential influence it has on the distribution of other diseases in one person. This information can provide a basis for selecting relevant comorbidities when designing clinical practice guidelines or deciding which comorbidity-based treatment recommendations are relevant.

The chronic condition with the highest number of associations was chronic low back pain, which also appeared to be by far the most important mediator of connections between other chronic conditions. High numbers of edges were also found in both genders for joint arthrosis and for several metabolic (hyperuricemia/gout, diabetes mellitus) and cardiovascular diseases (hypertension, chronic ischemic heart disease). The results presented here suggest that out of these conditions, hypertension is the one that serves as the most important bridge between other diseases. However, as our replication analysis shows, this result changes over time. In the 2004 data set, hyperuricemia/gout (males) and chronic ischemic heart diseases (females) seemed to serve as important bridges instead. Among women, depression was also characterized by many edges and it also seemed to be mediating many other disease connections. It should be noted that diseases with a higher prevalence did not necessarily have a higher degree centrality or betweenness centrality than diseases with a lower prevalence (e.g. in the male population, hyperuricemia/gout had only half the prevalence of lipid metabolism disorders but $50 \%$ more edges and a much higher betweenness centrality).

An association of low back pain with a large number of chronic conditions had been reported before, e.g. with cardiovascular diseases [16] as well as psychiatric diagnoses and pain syndromes [17]. In a previous study, we found that the prevalence of chronic low back pain increased more than expected by chance with the number 
Table 3 Triads with a prevalence $\geq 1 \%$ and an observed/expected ratio $\geq 2$ by multimorbidity clusters in the male population with $\geq 3$ chronic conditions $(n=54.987)$

O/E-ratio

Prevalence in \%

Obesity + Hyperuricemia/Gout + Liver diseases

Chronic ischemic heart disease + Cardiac arrhythmias + Cardiac insufficiency

Diabetes mellitus + Atherosclerosis/PAOD + Neuropathies

Chronic ischemic heart disease + Cardiac arrhythmias + Cardiac valve disorders

Diabetes mellitus + Obesity + Liver diseases

Severe vision reduction + Diabetes mellitus + Neuropathies

Chronic ischemic heart disease + Atherosclerosis/PAOD + Renal insufficiency

Chronic ischemic heart disease + Atherosclerosis/PAOD + Cardiac insufficiency

Diabetes mellitus + Atherosclerosis/PAOD + Renal insufficiency

Chronic ischemic heart disease + Hyperuricemia/Gout + Renal insufficiency

Chronic ischemic heart disease + Cardiac arrhythmias + Renal insufficiency

Chronic ischemic heart disease + Atherosclerosis/PAOD + Cerebral ischemia/Chronic stroke

Diabetes mellitus + Atherosclerosis/PAOD + Cerebral ischemia/Chronic stroke

Hypertension + Renal insufficiency + Cardiac insufficiency

Lipid metabolism disorders + Hyperuricemia/Gout + Liver diseases

Diabetes mellitus + Hyperuricemia/Gout + Liver diseases

Diabetes mellitus + Obesity + Hyperuricemia/Gout

Lipid metabolism disorders + Obesity + Liver diseases

Diabetes mellitus + Hyperuricemia/Gout + Renal insufficiency

Diabetes mellitus + Cardiac arrhythmias + Cardiac insufficiency

Hypertension + Urinary incontinence + Cancers

Lipid metabolism disorders + Hyperuricemia/Gout + Renal insufficiency

Diabetes mellitus + Chronic ischemic heart disease + Cardiac insufficiency

Hypertension + Cardiac arrhythmias + Cardiac valve disorders

Diabetes mellitus + Chronic ischemic heart disease + Renal insufficiency

Lipid metabolism disorders + Cardiac arrhythmias + Cardiac valve disorders

Chronic ischemic heart disease + Hyperuricemia/Gout + Cardiac insufficiency

Lipid metabolism disorders + Obesity + Hyperuricemia/Gout

Hypertension + Cardiac arrhythmias + Cardiac insufficiency

Diabetes mellitus + Chronic ischemic heart disease + Neuropathies

Chronic low back pain + Joint arthrosis + Hemorrhoids 
Table 3 Triads with a prevalence $\geq 1 \%$ and an observed/expected ratio $\geq 2$ by multimorbidity clusters in the male population with $\geq 3$ chronic conditions $(n=54.987)$ (Continued)

\begin{tabular}{llc}
\hline Chronic low back pain + Joint arthrosis + Insomnia & 2.1 & 1.3 \\
Chronic low back pain + Joint arthrosis + Severe hearing loss & 2.1 & 1.1 \\
Both multimorbidity patterns & O/E & 2.5 \\
Chronic ischemic heart disease + Asthma/COPD + Cardiac insufficiency & 2.2 & 1.0 \\
Chronic low back pain + Joint arthrosis + Neuropathies & 2.2 & 1.8 \\
Chronic low back pain + Obesity + Liver diseases & 2.1 & 1.4 \\
Chronic low back pain + Atherosclerosis/PAOD + Neuropathies & 2.0 & 1.1 \\
Joint arthrosis + Obesity + Hyperuricemia/Gout & 2.0 & 1.4 \\
Hyperuricemia/Gout + Liver diseases + Asthma/COPD & 2.0 & 1.1 \\
Hyperuricemia/Gout + Prostatic hyperplasia + Renal insufficiency & 1.1 \\
\hline O/E: observed/expected ratio; PAOD: peripheral arterial occlusive disease; COPD: chronic obstructive pulmonary disease; GERD: gastroesophageal reflux disease.
\end{tabular}

of comorbidities one person had [18]. For this reason, chronic low back pain might be more often the result of and not be the cause for other diseases. Low back pain could also be part of some general frailty pattern [16]. Raspe et al. suggest that back pain undergoes a unidirectional process of chronification towards a complex pain syndrome including other types of pain as well as functional and emotional impairments [19]. Another explanation might be that chronic low back pain is often found as a secondary diagnosis when multimorbid patients consult a physician for other reasons. E.g. Waxman et al. identified that $71 \%$ of patients with back pain did not see their physician for this reason and that depression symptoms had a higher impact on consultation rates for back pain than pain characteristics [20].

As stated above, we also detected a multitude of associations between disorders that belonged to the metabolic syndrome (i.e. obesity, hypertension, lipid metabolism disorders and diabetes mellitus [21]), hyperuricemia/gout and cardiovascular diseases (i.e. hypertension, atherosclerosis, chronic ischemic heart disease) in both genders. Such co-occurrences have been well documented. For example, the relative excess risk for cardiovascular disease is estimated to be two to eight times higher in people with diabetes than in non-diabetic individuals, when adjusted for age, sex, and ethnicity [22]. A main reason for this association might be an overlapping of behavioural and/or genetic risk factors for diabetes and cardiovascular disease [21]. It has also been implied that diabetes mellitus and gout share the same risk factors [23].

\section{Multimorbidity clusters}

Except for asthma/COPD, we found the same structure of multimorbidity clusters in the network analysis of triadic disease combinations in both genders as in the previously published factor analysis [9]. Reasons for these associations are discussed in the literature. For example, the association between respiratory and cardiovascular disease might be due to systemic inflammation, chronic infections, shared risk factors (such as smoking) or other undefined factors [24].

The network analysis also showed some associations within the multimorbidity clusters that were overlooked by factor analysis. Regarding network analysis, this relates particularly to neuropathies and severe vision reduction associated with the multimorbidity cluster of cardiovascular and metabolic disorders in both genders As both disorders are frequent complications of diabetes mellitus [25], this seems to be a plausible extension of this pattern. In females, the pattern of cardiovascular and metabolic disorders was amended by cerebral ischemia/chronic stroke, which had already been included in this pattern for the male population in the factor analysis.

We found three reasons for overlapping phenomena between the multimorbidity clusters cardiovascular and metabolic disorders and ADS and pain. First, there might be medical explanations, such as shared risk factors, causation and complications, e.g. the association between diseases that constitute the metabolic syndrome and musculoskeletal disorders. There is evidence that obesity and associated diseases, like diabetes mellitus, might be risk factors for low back pain $[26,27]$ and osteoarthritis $[28,29]$. There is also some evidence that anxiety and depression might lead to hypertension [30] and associated disorders. A second group of reasons for overlapping multimorbidity clusters might be related to utilization patterns of the different medical disciplines in ambulatory care. For example, an urologist consulted for prostatic hyperplasia might regularly test for renal insufficiency or hyperuricemia and, therefore, the prevalence of these diagnoses might be higher in patients with prostatic hyperplasia. Third, there is no obvious explanation for some associations between diseases of different patterns, e.g. the triad of cardiac valve disorders, cardiac arrhythmias and chronic low back pain. Research is needed to identify the mechanisms for this type of overlapping if these relations are confirmed. 
Table 4 Prevalence, number of edges, degree and betweenness centrality of diseases by gender and multimorbidity clusters in the population with $\geq \mathbf{3}$ chronic conditions

\begin{tabular}{|c|c|c|c|c|c|c|c|}
\hline \multirow[t]{2}{*}{ ADS and pain } & \multicolumn{3}{|c|}{ Prevalence in patients } & \multicolumn{2}{|c|}{ Number of edges (degree centrality) } & \multicolumn{2}{|c|}{ Betweenness centrality } \\
\hline & Total & Females & Males & Females & Males & Females & Males \\
\hline Chronic low back pain & $43.7 \%$ & $47.1 \%$ & $41.0 \%$ & $16(8.0 \%)$ & $16(9.2 \%)$ & $28.4 \%$ & $32.2 \%$ \\
\hline Joint arthrosis & $28.7 \%$ & $33.6 \%$ & $24.8 \%$ & $10(5.0 \%)$ & $9(5.2 \%)$ & $4.9 \%$ & $5.7 \%$ \\
\hline Lower limb varicosis & $16.4 \%$ & $22.7 \%$ & $11.5 \%$ & $7(3.5 \%)$ & $2(1.1 \%)$ & $3.1 \%$ & $0.0 \%$ \\
\hline Prostatic hyperplasia & * & - & $28.1 \%$ & - & $5(2.9 \%)$ & - & $2.6 \%$ \\
\hline Asthma/COPD & $15.3 \%$ & $14.1 \%$ & $16.3 \%$ & $3(1.5 \%)$ & $4(2.3 \%)$ & $0.4 \%$ & $0.1 \%$ \\
\hline Depression & $12.4 \%$ & $18.1 \%$ & $7.8 \%$ & $10(5.0 \%)$ & $2(1.1 \%)$ & $11.9 \%$ & $0.0 \%$ \\
\hline Chronic gastritis/GERD & $10.8 \%$ & $10.8 \%$ & $10.8 \%$ & $7(3.5 \%)$ & $3(1.7 \%)$ & $2.7 \%$ & $0.1 \%$ \\
\hline Osteoporosis & $10.7 \%$ & $19.7 \%$ & $3.6 \%$ & $2(1.0 \%)$ & - & $0.0 \%$ & - \\
\hline Gynaecological problems & * & $16.6 \%$ & - & $3(1.5 \%)$ & - & $0.1 \%$ & - \\
\hline Allergies & $7.3 \%$ & $8.5 \%$ & $6.3 \%$ & $3(1.5 \%)$ & - & $0.4 \%$ & - \\
\hline Insomnia & $6.6 \%$ & $7.6 \%$ & $5.8 \%$ & $4(2.0 \%)$ & $2(1.1 \%)$ & $0.2 \%$ & $0.0 \%$ \\
\hline Intestinal diverticulosis & $6.3 \%$ & $6.4 \%$ & $6.2 \%$ & $2(1.0 \%)$ & $2(1.1 \%)$ & $0.0 \%$ & $0.0 \%$ \\
\hline Hemorrhoids & $6.1 \%$ & $5.2 \%$ & $6.8 \%$ & $2(1.0 \%)$ & $4(2.3 \%)$ & $0.0 \%$ & $0.4 \%$ \\
\hline Somatoform disorders & $5.9 \%$ & $7.7 \%$ & $4.5 \%$ & $5(2.5 \%)$ & - & $1.1 \%$ & - \\
\hline Severe hearing loss & $4.6 \%$ & $3.8 \%$ & $5.2 \%$ & - & $2(1.1 \%)$ & - & $0.0 \%$ \\
\hline Dizziness & $4.2 \%$ & $5.4 \%$ & $3.4 \%$ & $2(1.0 \%)$ & - & $0.0 \%$ & - \\
\hline Anxiety & $2.5 \%$ & $3.7 \%$ & $1.6 \%$ & $2(1.0 \%)$ & - & $0.0 \%$ & - \\
\hline Sexual dysfunction & $*$ & - & $4.5 \%$ & - & $2(1.1 \%)$ & - & $0.0 \%$ \\
\hline \multicolumn{8}{|l|}{ Cardiovascular and metabolic } \\
\hline Hypertension & $69.3 \%$ & $69.3 \%$ & $69.4 \%$ & $13(6.5 \%)$ & $9(5.2 \%)$ & $12.0 \%$ & $12.0 \%$ \\
\hline Lipid metabolism disorders & $47.4 \%$ & $46.7 \%$ & $48.0 \%$ & $11(5.5 \%)$ & $8(4.6 \%)$ & $5.8 \%$ & $1.4 \%$ \\
\hline Diabetes mellitus & $28.5 \%$ & $24.9 \%$ & $31.4 \%$ & $14(7.0 \%)$ & $11(6.3 \%)$ & $5.8 \%$ & $5.0 \%$ \\
\hline Chronic ischemic heart disease & $25.7 \%$ & $18.2 \%$ & $31.6 \%$ & $13(6.5 \%)$ & $10(5.7 \%)$ & $5.1 \%$ & $2.8 \%$ \\
\hline Hyperuricemia/Gout & $18.1 \%$ & $11.3 \%$ & $23.5 \%$ & $11(5.5 \%)$ & $12(6.9 \%)$ & $5.2 \%$ & $7.8 \%$ \\
\hline Cardiac arrhythmias & $18.0 \%$ & $15.5 \%$ & $20.0 \%$ & $6(3.0 \%)$ & $8(4.6 \%)$ & $0.2 \%$ & $4.3 \%$ \\
\hline Atherosclerosis/PAOD & $13.7 \%$ & $10.3 \%$ & $16.4 \%$ & $8(4.0 \%)$ & $10(5.7 \%)$ & $2.6 \%$ & $5.7 \%$ \\
\hline Obesity & $12.4 \%$ & $13.4 \%$ & $11.7 \%$ & $9(4.5 \%)$ & $7(4.0 \%)$ & $2.2 \%$ & $3.8 \%$ \\
\hline Liver diseases & $12.0 \%$ & $9.9 \%$ & $13.7 \%$ & $7(3.5 \%)$ & $8(4.6 \%)$ & $2.0 \%$ & $4.5 \%$ \\
\hline Cardiac insufficiency & $9.0 \%$ & $10.1 \%$ & $8.1 \%$ & $8(4.0 \%)$ & $8(4.6 \%)$ & $1.0 \%$ & $1.3 \%$ \\
\hline Cerebral ischemia/Chronic stroke & $9.0 \%$ & $7.3 \%$ & $10.3 \%$ & $2(1.0 \%)$ & $4(2.3 \%)$ & $0.0 \%$ & $0.1 \%$ \\
\hline Neuropathies & $8.2 \%$ & $8.1 \%$ & $8.2 \%$ & $7(3.5 \%)$ & $6(3.4 \%)$ & $4.6 \%$ & $3.9 \%$ \\
\hline Renal insufficiency & $6.5 \%$ & $4.2 \%$ & $8.3 \%$ & $4(2.0 \%)$ & $9(5.2 \%)$ & $0.0 \%$ & $2.6 \%$ \\
\hline Cardiac valve disorders & $5.7 \%$ & $5.2 \%$ & $6.0 \%$ & $4(2.0 \%)$ & $5(2.9 \%)$ & $0.1 \%$ & $2.2 \%$ \\
\hline \multicolumn{8}{|l|}{ No multimorbidity pattern } \\
\hline Severe vision reduction & $20.6 \%$ & $22.0 \%$ & $19.6 \%$ & $3(1.5 \%)$ & $2(1.1 \%)$ & $0.1 \%$ & $0.0 \%$ \\
\hline Cancers & $18.4 \%$ & $14.8 \%$ & $21.2 \%$ & - & $2(1.1 \%)$ & - & $0.0 \%$ \\
\hline Urinary incontinence & $5.1 \%$ & $7.1 \%$ & $3.5 \%$ & $2(1.0 \%)$ & $2(1.1 \%)$ & $0.0 \%$ & $0.0 \%$ \\
\hline
\end{tabular}

ADS: Anxiety, depression and somatoform disorders; PAOD: peripheral arterial occlusive disease; COPD: chronic obstructive pulmonary disease; GERD: gastro-esophageal reflux disease; * gender-specific disease.

\section{Gender differences}

Diseases seem to be more associated to each other in the female population (with 15\% more edges and a slightly lower average node betweenness) than in the male population.
This phenomenon can be partly explained by an $11 \%$ higher number of triads with a prevalence $\geq 1 \%$ in females than in males. However, we generally found a comparable disease network in both genders. In the cluster of cardiovascular 


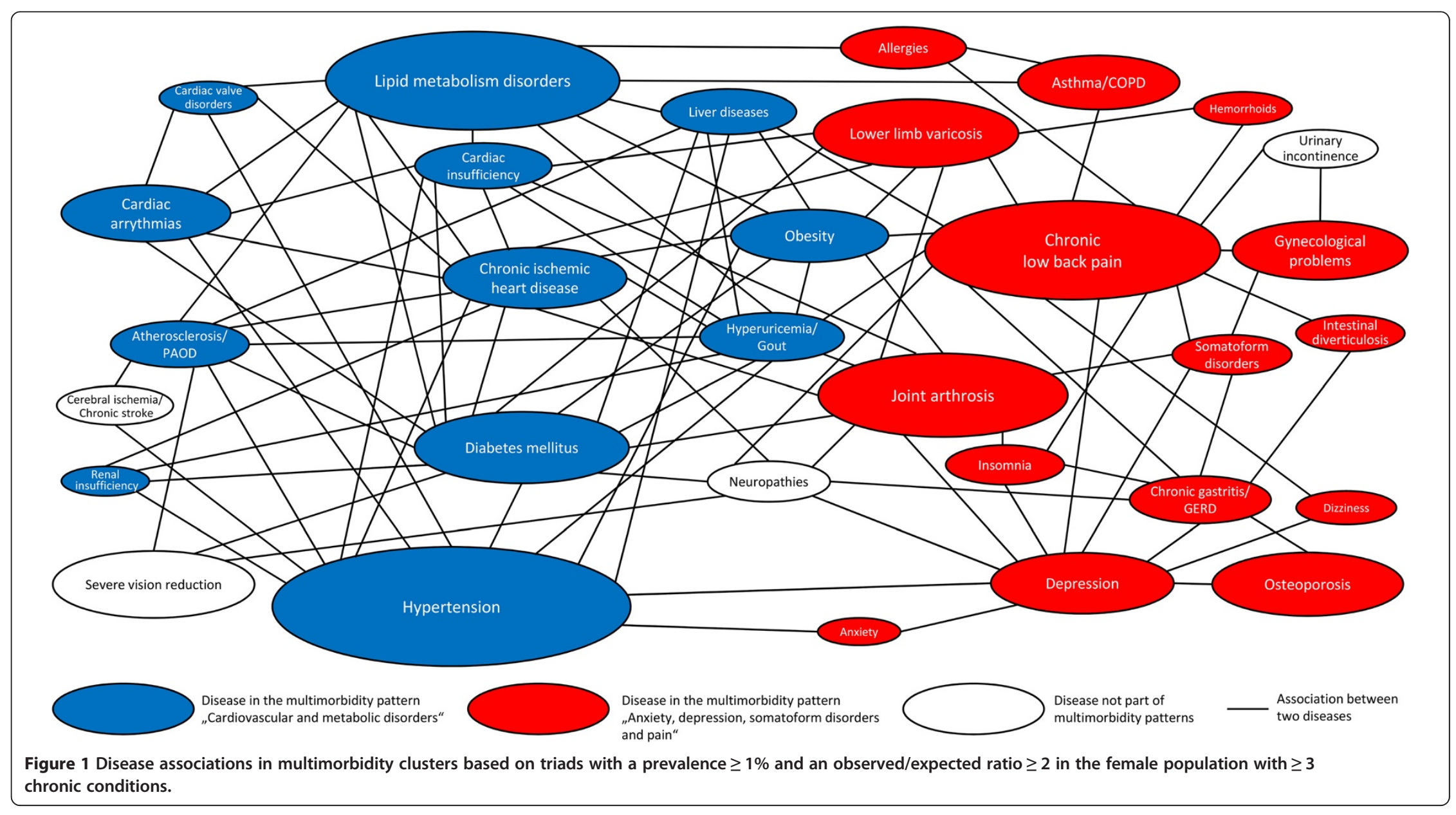




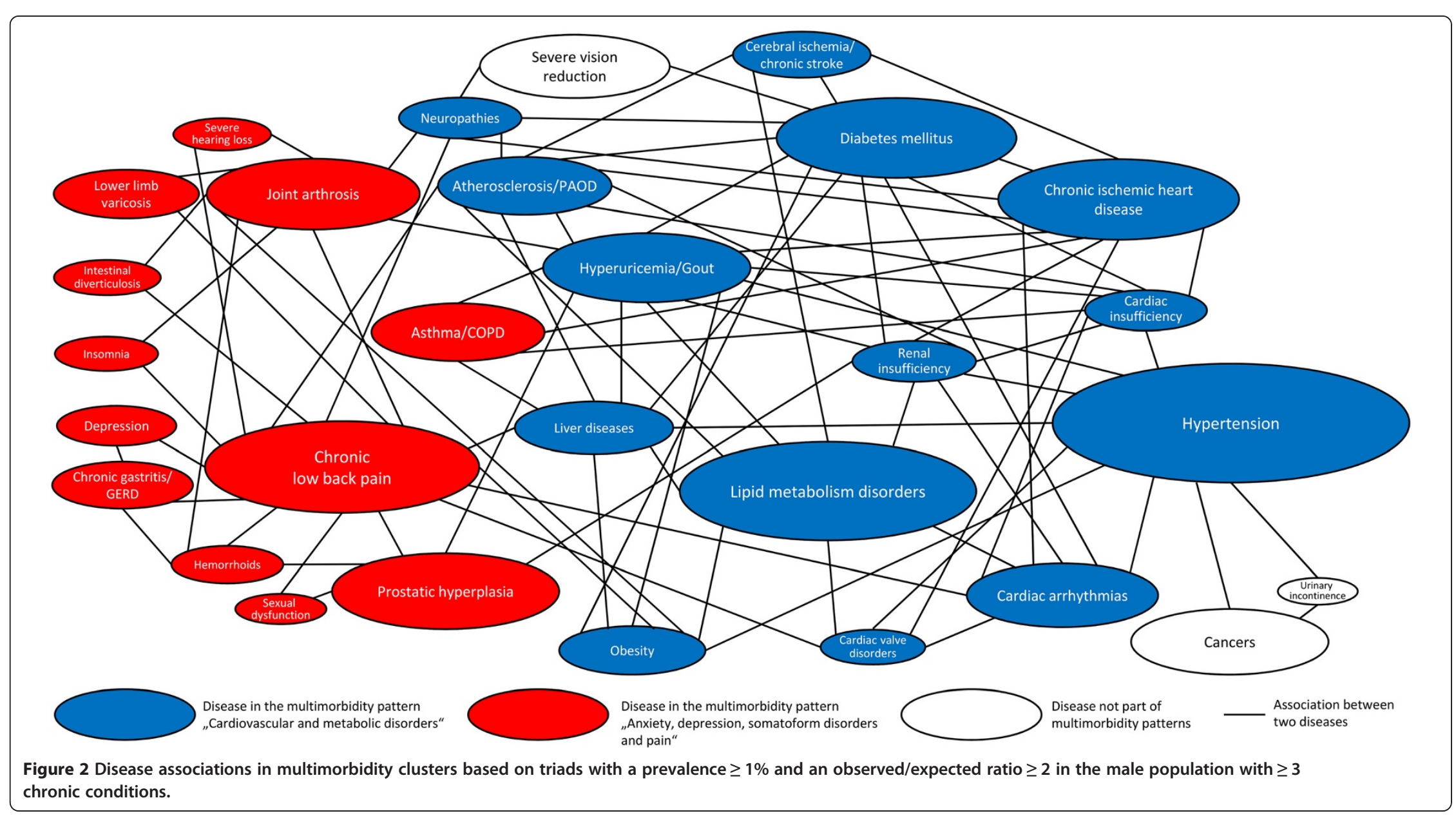


and metabolic disorders, we found a high concordance between both genders. Females and males both had diabetes mellitus, chronic ischemic heart disease and hyperuricemia/ gout among the five most associated diseases and comparable disease associations in this cluster.

Gender differences were more prominent in the multimorbidity cluster of ADS and pain. Although chronic low back pain and joint arthrosis were the two most associated diseases in both genders, women generally had more associations with psychiatric/psychosomatic disorders, e.g. the diagnoses anxiety and somatoform disorders were only present in the disease matrix of the female population. This also applied for cases of depression which had a comparably high degree and betweenness centrality in females, but not in males. The main reason for these differences might be due to gender-dependent prevalence rates, e.g. the prevalence of depression was more than twice as high in the female than in the male population in our study. These gender-differences related to depression are confirmed by a wide range of other studies [31]. Although sometimes influenced by artefacts, like reporting bias, genderdifferences in depression can be considered to be a genuine phenomenon [21].

\section{Strengths and weaknesses}

This study used a network analysis to combine results from factor analysis and observed/expected ratios of disease combinations in order to create a pattern-specific disease network. The methods were well matched as multimorbidity clusters from factor analysis could also be found in the network analysis and some diseases were identified that had been overlooked by factor analysis. The figures provided a systematic overview of all disease associations that were prevalent and more frequent than expected by chance. The analyses were based on triads of diseases because multimorbidity was defined by a three disease criterion. For this reason, associations that occurred only between two diseases were not considered.

A strength of our approach lies in the comprehensive selection of diseases by including all highly prevalent chronic conditions $(\geq 1 \%$ in the age group $65+)$ into our diagnosis groups and applying them to our disease matrix. Unfortunately, a more recent data set than 2006 was not available for these analyses. However, we assume that the associations between diseases that we found are, for the most part, stable over time, thus results based on newer data will probably be similar. To provide more confidence in our results, we did a replication analysis with the 2004 data set of the same insurance company, which used the same methods as our 2006 analyses, except for a lowered prevalence criterion for triad inclusion. The reason for this decision was a much higher prevalence of the most chronic conditions in 2006 compared to 2004, probably due to changes in the documentation behaviour of ambulatory physicians. This phenomenon had been reported before, e.g. Uijen and Lisdonk found a $60 \%$ increase in the number of diagnoses of chronic conditions in the Netherlands between 1985 and 2005 [32]. This trend could probably also be observed if a more recent data set was used, which might mean that the prevalence criterion for triad inclusion needed to be increased in later years in order to keep results comparable. Our replication analysis confirmed the results presented here. However, there are generally great differences concerning the identification of multimorbidity clusters in different studies [8], so that there is still need for replication in other countries and populations.

It should also be noted that the study is based on diagnoses and not on diseases. Although accidental and transitory diagnoses were excluded, in some cases, diagnoses might be imprecise, ambiguous, or incomplete because they were not clinically verified by trained professionals. This is a general problem with insurance claims data [33], but in our view, the benefits of claims data outweigh their disadvantages: We are provided with a large unselected population group, representing real-world conditions, including people living in protected institutions/nursing homes, as well as frail individuals, and the oldest-old, i.e. groups of patients frequently not included in survey and field studies. In choosing insurance claims data, we also avoided selection bias concerning service providers, and there is no recall bias concerning diagnosis data. However, as results from multimorbidity studies might differ depending on the data set used [34], a replication of our study using other data sources should also be useful.

\section{Conclusion}

Using the current methods for analysing associations between diseases, we knew the clustering of diseases and frequent disease combinations, but we missed the complete picture of multimorbidity, because the internal structure of the clusters and the linking between disease combinations and clusters were still unknown. The results presented here constitute the first attempt to respond to this academic vault. We are able to show which diseases are associated with each other in our data set, to which multimorbidity clusters the diseases are assigned and which diseases are responsible for overlapping multimorbidity clusters.

However, we have to keep in mind that this approach focuses on statistically selected diseases and that most patients suffer from more diseases than those represented in our analyses. For this reason, multimorbidity cannot be grasped completely. Nevertheless, our methodological approach might prove a good starting point for further analyses and guideline developers might find our approach useful as a basis for discussing which comorbidity should be addressed in clinical practice guidelines. 


\section{Competing interests}

The authors declare that they have no competing interests.

\section{Authors' contributions}

HvdB, IS, HK and MS conceived and designed the study. IS and GS analysed the data. IS drafted the manuscript. HK and HOW participated in clinical discussions of study results. All authors read and approved the final manuscript.

\section{Acknowledgements}

We thank the health insurance company BARMER/GEK for providing the data. The study was funded by the German Federal Ministry of Education and Research (http://www.bmbf.bund.de/; grant numbers 01ET0725, 01 ET0731 and 01ET1006A-K). The funders had no role in study design, data collection and analysis, decision to publish or preparation of the manuscript.

\section{List of frequently used terms}

Betweenness centrality: Measure for the potential influence of a disease on the distribution of other diseases in one person. Defined as the number of times a disease serves as a bridge in the shortest pathway between two other diseases in relation to the total number of diseases in the network. Bridge: Mediator between two diseases. Defined as a disease that has to be crossed to get in the shortest pathway from one disease to another disease if there is no direct association between these diseases.

Degree centrality: Measure for the connectedness of a disease. Defined as the number of associations of one disease with other diseases in relation to the total number of diseases in the network.

Edge: Disease pair/association between two diseases. Diseases are defined to be associated if they are part of a disease triad with a prevalence $\geq 1 \%$ and an observed/expected-ratio is $\geq 2$.

Edgelist: A list of all disease pairs contained within the selected triads. Multimorbidity cluster: Group of diseases that often occur together. Multimorbidity clusters are determined by tetrachoric factor analysis. Node: Element of the network analysis. In the analyses presented here, diseases are used as nodes.

Observed/expected ratio: Measure for the excess prevalence of a disease combination which is used to determine if there is an association between diseases (in case the ratio is $\geq 2$ ). Defined as the ratio between the prevalence of a disease combination and the product of the prevalences of all single diseases within this combination.

Triad: Combination of three diseases found in the data set.

\section{Author details}

${ }^{1}$ Department of Primary Medical Care, University Medical Center Hamburg-Eppendorf, Martinistr. 52, Hamburg 20246, Germany. ${ }^{2}$ Institute of General Practice, Medical Faculty, University of Kiel, Kiel, Germany. ${ }^{3}$ Department of Medical Biometry and Epidemiology, University Medical Center Hamburg-Eppendorf, Hamburg, Germany.

Received: 21 July 2014 Accepted: 10 December 2014 Published: 16 December 2014

\section{References}

1. van den Bussche H, Hansen H, Koller D, Kolonko T, Wegscheider K, Glaeske G, von Leitner EC, Schäfer I, Schön G: Which chronic diseases and disease patterns are specific for multimorbidity in the elderly? Results of a claims data based cross-sectional study in Germany. BMC Public Health 2011, 11:101.

2. Fortin M, Bravo G, Hudon C, Vanasse A, Lapointe L: Prevalence of multimorbidity among adults seen in family practice. Ann Fam Med 2005, 3:223-228.

3. Gijsen R, Hoeymans N, Schellevis FG, Ruwaard D, van den Satariano WA, Bos GA: Causes and consequences of comorbidity a review. J Clin Epidemiol 2001, 54:661-674.

4. Marengoni A, Angleman S, Melis R, Mangialasche F, Karp A, Garmen A, Meinow B, Fratiglioni L: Aging with multimorbidity: a systematic review of the literature. Ageing Res Rev 2011, 10:430-439.

5. Marengoni A, Rizzuto D, Wang HX, Winblad B, Fratiglioni L: Patterns of chronic multimorbidity in the elderly population. J Am Geriatr Soc 2009, 57:225-230.
6. Holden L, Scuffham PA, Hilton MF, Muspratt A, Ng SK, Whiteford HA: Patterns of multimorbidity in working Australians. Population Health Metrics 2011, 9:15.

7. Wiesner G, Grimm J, Bittner E: Multimorbidität in Deutschland. Stand Entwicklung - Folgen. Berlin: Robert-Koch-Institut; 2003.

8. Prados-Torres A, Calderón-Larrañaga A, Hancco-Saavedra J, Poblador-Plou B, van den Akker M: Multimorbidity patterns: a systematic review. J Clin Epidemiol 2014, 67:254-266.

9. Schäfer I, von Leitner EC, Schön G, Koller D, Hansen H, Kolonko T, Kaduszkiewicz H, Wegscheider K, Glaeske G, van den Bussche H: Multimorbidity patterns in the elderly: A new approach of disease clustering identifies complex interrelations between chronic conditions. PLOS One 2010, 5:e15941.

10. Kassenärztliche Bundesvereinigung: Grunddaten zur vertragsärztlichen Versorgung. 2006, http://www.kbv.de/html/421.php

11. Kassenärztliche Bundesvereinigung: Bundesmantelverträge. http://www.kbv. de/html/bundesmantelvertrag.php.

12. Zentralinstitut für die kassenärztliche Versorgung: Daten des ADT-Panels des Zentralinstituts für die kassenärztliche Versorgung. http://www.gbe-bund.de.

13. Busse R, Drösler S, Glaeske G, Greiner W, Schäfer T, Schrappe M: Wissenschaftliches Gutachten für die Auswahl von 50 bis 80 Krankheiten zur Berücksichtigung im morbiditätsorientierten Risikostrukturausgleich. 2007, Bonn: Wissenschaftlicher Beirat des Bundesversicherungsamtes; 2007.

14. Corten R: Visualization of social networks in Stata using multidimensional scaling. Stata Journal 2011, 11(1):52-63.

15. R Core Team: R. A Language and Environment for Statistical Computing. Vienna, Austria: R Foundation for Statistical Computing; 2013.

16. Hestbaek L, Leboeuf-Yde C, Manniche C: Is low back pain part of a general health pattern or is it a separate and distinctive entity? A critical literature review of comorbidity with low back pain. J Manipulative Physiol Ther 2003, 26:243-252.

17. Hagen EM, Svensen E, Eriksen HR, Ihlebaek CM, Ursin H: Comorbid subjective health complaints in low back pain. Spine 2006, 31:1491-1495.

18. Schäfer I: Does multimorbidity influence the occurrence rates of chronic conditions? a claims data based comparison of expected and observed prevalence rates. PLoS One 2012, 7:e45390.

19. Raspe H, Hüppe A, Matthis C: Theories and models of chronicity: on the way to a broader definition of chronic back pain. Schmerz 2003, 17:359-366.

20. Waxman $R$, Tennant A, Helliwell P: Community survey of factors associated with consultation for low back pain. BMJ 1998, 317:1564.

21. Alberti KG, Zimmet P, Shaw J: Metabolic syndrome - a new world-wide definition. A Consensus Statement from the International Diabetes Federation. Diabet Med 2006, 23:469-480.

22. Howard BV, Rodriguez BL, Bennett PH, Harris MI, Hamman R, Kuller LH, Pearson TA, Wylie-Rosett J: Prevention conference VI: diabetes and cardiovascular disease: writing group I: epidemiology. Circulation 2002, 105:e132-e137.

23. Choi HK, De Vera MA, Krishnan E: Gout and the risk of type 2 diabetes among men with a high cardiovascular risk profile. Rheumatology 2008, 47:1567-1570.

24. Mannino DM, Thorn D, Swensen A, Holguin F: Prevalence and outcomes of diabetes, hypertension and cardiovascular disease in COPD. Eur Respir J 2008, 32:962-969.

25. Deshpande AD, Harris-Hayes M, Schootman M: Epidemiology of diabetes and diabetes-related complications. Phys Ther 2008, 88:1254-1264.

26. Shiri R, Karppinen J, Leino-Arjas P, Solovieva S, Viikari-Juntura E: The association between obesity and Low back pain: a meta-analysis. Am J Epidemiol 2010, 171:135-154.

27. Manchikanti L: Epidemiology of Low back pain. Pain Physician 2000, 3:167-192.

28. Carman WJ, Sowers MF, Hawthorne VM, Weissfeld LA: Obesity as a risk factor for osteoarthritis of the hand and wrist: a prospective study. Am J Epidemiol 1994, 139:119-129.

29. Stürmer T, Brenner H, Brenner RE, Günther KP: Non-insulin dependent diabetes mellitus (NIDDM) and patterns of osteoarthritis: the Ulm osteoarthritis study. Scand J Rheumatol 2001, 30:169-171.

30. Jonas BS, Franks P, Ingram DD: Are symptoms of anxiety and depression risk factors for hypertension? Longitudinal evidence from the National Health and Nutrition Examination Survey I Epidemiologic Follow-up Study. Arch Fam Med 1997, 6:43. 
31. Piccinelli M, Wilkinson G: Gender differences in depression. Critical review. Brit J Psychiat 2000, 177:486-492.

32. Uijen $A A$, van de Lisdonk EH: Multimorbidity in primary care. Prevalence and trend over the last 20 years. Eur J Gen Pract 2008, 14(S1):28-32.

33. Erler A, Beyer M, Muth C, Gerlach FM, Brennecke R: Garbage in - garbage out? validity of coded diagnoses from GP claims records. Gesundheitswesen 2009, 71:823-831.

34. van den Bussche H, Schäfer I, Wiese B, Dahlhaus A, Fuchs A, Gensichen J, Höfels S, Hansen H, Leicht H, Koller D, Luppa M, Nützel A, Werle J, Scherer M, Wegscheider K, Glaeske G, Schön G: A comparative study demonstrated that prevalence figures on multimorbidity require cautious interpretation when drawn from a single database. J Clin Epidemiol 2013, 66:209-217.

doi:10.1186/1471-2458-14-1285

Cite this article as: Schäfer et al:: Reducing complexity: a visualisation

of multimorbidity by combining disease clusters and triads. BMC Public Health 2014 14:1285.

\section{Submit your next manuscript to BioMed Central and take full advantage of:}

- Convenient online submission

- Thorough peer review

- No space constraints or color figure charges

- Immediate publication on acceptance

- Inclusion in PubMed, CAS, Scopus and Google Scholar

- Research which is freely available for redistribution 